\title{
On reducing inconsistency of pairwise comparison matrices below an acceptance threshold
}

\author{
Sándor BOZÓKI ${ }^{1}$ \\ Laboratory on Engineering and Management Intelligence, \\ Research Group of Operations Research and Decision Systems, \\ Institute for Computer Science and Control, \\ Hungarian Academy of Sciences (MTA SZTAKI); and \\ Department of Operations Research and Actuarial Sciences \\ Corvinus University of Budapest, Hungary \\ bozoki.sandor@sztaki.mta.hu \\ János FÜLÖP \\ Laboratory on Engineering and Management Intelligence, \\ Research Group of Operations Research and Decision Systems, \\ Institute for Computer Science and Control, \\ Hungarian Academy of Sciences (MTA SZTAKI); Budapest, Hungary \\ fulop.janos@sztaki.mta.hu \\ Attila POESZ \\ Department of Operations Research and Actuarial Sciences \\ Corvinus University of Budapest, Hungary \\ attila.poesz@uni-corvinus.hu
}

4 November 2013

Research was supported in part by OTKA grant K77420.

\footnotetext{
${ }^{1}$ corresponding author
} 


\begin{abstract}
A recent work of the authors on the analysis of pairwise comparison matrices that can be made consistent by the modification of a few elements is continued and extended. Inconsistency indices are defined for indicating the overall quality of a pairwise comparison matrix. It is expected that serious contradictions in the matrix imply high inconsistency and vice versa. However, in the 35-year history of the applications of pairwise comparison matrices, only one of the indices, namely $C R$ proposed by Saaty, has been associated to a general level of acceptance, by the well known ten percent rule. In the paper, we consider a wide class of inconsistency indices, including $C R, C M$ proposed by Koczkodaj and Duszak and $C I$ by Peláez and Lamata. Assume that a threshold of acceptable inconsistency is given (for $C R$ it can be 0.1 ). The aim is to find the minimal number of matrix elements, the appropriate modification of which makes the matrix acceptable. On the other hand, given the maximal number of modifiable matrix elements, the aim is to find the minimal level of inconsistency that can be achieved. In both cases the solution is derived from a nonlinear mixed-integer optimization problem. Results are applicable in decision support systems that allow real time interaction with the decision maker in order to review pairwise comparison matrices.
\end{abstract}

Keywords: Multi-attribute decision making, pairwise comparison matrix, inconsistency, mixed 0-1 convex programming

\title{
1 Introduction
}

Pairwise comparison matrices (Saaty, 1977) are used in multi-attribute decision problems, where relative importance of the criteria, the evaluations of the alternatives with respect to each criterion are to be quantified. The method of pairwise comparison is also applied for determining voting powers in group decision making. One of the advantages of pairwise comparison matrices is that the decision maker is faced to a sequence of elementary questions concerning the comparison of two criteria/alternatives at a time, instead of a complex task of providing the weights of the whole set of them.

A real $n \times n$ matrix $A$ is a pairwise comparison matrix if it is positive and reciprocal, i.e.,

$$
\begin{aligned}
a_{i j} & >0, \\
a_{i j} & =\frac{1}{a_{j i}}
\end{aligned}
$$


for all $i, j=1, \ldots, n$. A is consistent if the transitivity property

$$
a_{i j} a_{j k}=a_{i k}
$$

holds for all $i, j, k=1,2, \ldots, n$; otherwise it is called inconsistent.

For a positive $n \times n$ matrix $A$, let $\bar{A}=\log A$ denote the $n \times n$ matrix with the elements

$$
\bar{a}_{i j}=\log a_{i j}, \quad i, j=1, \ldots, n .
$$

Then $A$ is consistent if and only if

$$
\bar{a}_{i j}+\bar{a}_{j k}+\bar{a}_{k i}=0, \forall i, j, k=1, \ldots, n
$$

holds. Matrices $\bar{A}$ fulfilling the homogenous linear system (4) constitute a linear subspace in $\mathbb{R}^{n \times n}$.

Let $\mathcal{P}_{n}$ denote the set of the $n \times n$ pairwise comparison matrices, and $\mathcal{C}_{n} \subset \mathcal{P}_{n}$ the set of the consistent matrices. Since the reciprocity constraint (2) corresponds to $\bar{a}_{i j}=-\bar{a}_{j i}$ in the logarithmized space, the set $\log \mathcal{P}_{n}=$ $\left\{\log A \mid A \in \mathcal{P}_{n}\right\}$ is the set of $n \times n$ skew-symmetric matrices, an $n(n-1) / 2$ dimensional linear subspace of $\mathbb{R}^{n \times n}$. The set $\log \mathcal{C}_{n}=\left\{\log A \mid A \in \mathcal{C}_{n}\right\}$ is the set of matrices fulfilling (4), and as pointed out in Chu (1997), is an $(n-1)$-dimensional linear subspace of $\mathbb{R}^{n \times n}$. Clearly, $\log \mathcal{C}_{n} \subset \log \mathcal{P}_{n}$.

In decision problems of real life, the pairwise comparison matrices are rarely consistent. Nevertheless, decision makers are interested in the level of inconsistency of their judgements, which somehow expresses the goodness or "quality" of pairwise comparisons totally, because conflicting judgements may lead to senseless decisions. Therefore, some index is needed to measure the possible contradictions and inconsistencies of the pairwise comparison matrix.

A function $\phi_{n}: \mathcal{P}_{n} \rightarrow R$ is called an inconsistency index if $\phi_{n}(A)=0$ for every consistent and $\phi_{n}(A)>0$ for every inconsistent pairwise comparison matrix $A$. The inconsistency indices used in the practice are continuous, and the value of $\phi_{n}(A)>0$ indicates, more or less, how much an inconsistent matrix differs from a consistent one.

Since in the practice the consistency of a pairwise comparison matrix is not easy to assure, certain level of inconsistency is usually accepted by the decision makers. This works in the practice in such a way that for a given inconsistency index $\phi_{n}$ an acceptance threshold $\alpha_{n} \geq 0$ is chosen, and a matrix $A \in \mathcal{P}_{n}$ is kept for further use only if $\phi_{n}(A) \leq \alpha_{n}$ holds; otherwise, it is rejected or the pairwise comparisons are carried out again. The carrying out of all pairwise comparisons for filling-in the matrix is often a time-consuming task. Therefore, before the total rejection of a pairwise comparison matrix 
with an inconsistency level above a prescribes acceptance threshold, it may be worth investigating whether it is possible to improve the inconsistency of the matrix to an acceptable level by performing fewer pairwise comparisons.

The paper will concentrate on the following problem: for a given $A \in \mathcal{P}_{n}$, inconsistency index $\phi_{n}$ and acceptance level $\alpha_{n}$, what is the minimal number of the elements of matrix $A$ that by modifying these elements, and of course their reciprocals, the pairwise comparison matrix can be made acceptable. We shall show that under a slight boundedness assumption, this can be achieved by solving a nonlinear mixed 0-1 optimization problem. If it comes out that the matrix can be turned into an acceptable one by modifying relatively few elements, then it may be a case when a more-or-less consistent evaluator was less attentive at these few elements, or a data-recording error happened. So it may be worth re-evaluating these elements. Of course, if the the evaluator insists on the previous values, or the acceptable inconsistency threshold cannot be reached with the new values, then this approach was unsuccessful: all pairwise comparisons are to be evaluated again. If however after the revision of the critical elements, the inconsistency level of the modified matrix is already acceptable, then we can continue the decision process with it.

Concerning the investigations above, when solving the nonlinear mixed 0-1 programming problems, it is very beneficial if the nonlinear optimization problems obtained after the relaxation of the 0-1 variables are convex optimization problems. In the convex case several sophisticated methods and softwares are available, while in the nonconvex case methodological and implementation difficulties may arise. Since $\log \mathcal{C}_{n}$ is a linear subspace, $\mathcal{C}_{n}$ is a nonconvex manifold in $\mathbb{R}^{n \times n}$. One can immediately conclude that it is better to investigate the convexity issues in the logarithmized space.

Several proposals of inconsistency indices are known, see the overviews of Brunelli and Fedrizzi (2011, 2013a) and Brunelli et al. (2013b) for detailed lists and properties. This paper focuses on three well-known inconsistency indices. They are $C R$ proposed by Saaty (1980), $C M$ proposed by Koczkodaj and Duszak (Koczkodaj 1993; Duszak and Koczkodaj 1994), and CI proposed by Peláez and Lamata (2003). The properties and relationship of the fundamental indices $C R$ and $C M$ were also studied in Bozóki and Rapcsák (2008). In this paper we point out that for the inconsistency indices in our focus, the nonlinear mixed 0-1 optimization problems mentioned above can be formulated in the logarithmized space, and appropriate convexity properties hold on them. We show that $C R$ and $C I$ are convex function in the logarithmized space, and $C M$ is quasiconvex, but can be transformed into a convex function by applying a suitable strictly monotone univariate function on it. 
This paper is in a close relation to an earlier paper of the authors (Bozóki et al. 2011b). In the latter paper we investigated the special case when the acceptance threshold $\alpha_{n}$ is 0 , i.e. the modified pairwise comparison matrix must be consistent. No inconsistency indices were needed for this investigation, simple graph theoretic ideas were applied. Unfortunately, the technique applied for $\alpha_{n}=0$ cannot be extended to the general case, therefore, a new approach is proposed in this paper.

We also mention that some of the issues investigated in this paper were already considered, in Hungarian, in Bozóki et al. (2012).

Since inconsistent matrices are in the focus of this paper, and for $n=1$ and $n=2$ the pairwise comparison matrices are consistent, we shall assume in the sequel, without loss of generality, that $n \geq 3$.

In Section 2, the optimization problems to be solved are presented in a general form. The general issues are specialized and investigated for the inconsistency indices $C R$ of Saaty, $C M$ of Koczkodaj and Duszak, and $C I$ proposed by Peláez and Lamata in Sections 3 through 5, respectively. A numerical example is presented in Section 6.

\section{The general form of the optimization prob- lems}

Let $\phi_{n}$ be an inconsistency index and $\alpha_{n}$ be an acceptance threshold, and let

$$
\mathcal{A}_{n}\left(\phi_{n}, \alpha_{n}\right)=\left\{A \in \mathcal{P}_{n} \mid \phi_{n}(A) \leq \alpha_{n}\right\}
$$

denote the set of $n \times n$ pairwise comparison matrices with inconsistency $\phi_{n}$ not exceeding threshold $\alpha_{n}$. Let $A, \hat{A} \in \mathcal{P}_{n}$ and

$$
d(A, \hat{A})=\left|\left\{(i, j): 1 \leq i<j \leq n, a_{i j} \neq \hat{a}_{i j}\right\}\right|
$$

denote the number of matrix elements above the main diagonal, where matrices $A$ and $\hat{A}$ differ from each other. By reciprocity, the number of different elements is the same as in positions below the main diagonal.

Consider pairwise comparison matrix $A \in \mathcal{P}_{n}$ with $\phi_{n}(A)>\alpha_{n}$ as it is not acceptable in terms of inconsistency. We want to calculate the minimal number of matrix elements above the main diagonal to be modified in order to make matrix acceptable (elements below the main diagonal are determined by the elements above the main diagonal). That is to solve the optimization problem

$$
\begin{array}{ll}
\min & d(A, \hat{A}) \\
\text { s.t. } & \hat{A} \in \mathcal{A}_{n}\left(\phi_{n}, \alpha_{n}\right),
\end{array}
$$


where the elements above the main diagonal of $\hat{A}$ are variables.

We could also ask the minimal inconsistency of $A \in \mathcal{P}_{n}$ matrix can be reached by modifying at most $K$ elements and their reciprocals. The optimization problem is

$$
\begin{array}{ll}
\min & \alpha \\
\text { s.t. } & d(A, \hat{A}) \leq K, \\
& \hat{A} \in \mathcal{A}_{n}\left(\phi_{n}, \alpha\right),
\end{array}
$$

where $\alpha$ and the elements above the main diagonal of $\hat{A}$ are variables.

Problems (77) and (8) can be formulated in logarithmic space:

$$
\log \mathcal{A}_{n}\left(\phi_{n}, \alpha_{n}\right)=\left\{X \in \log \mathcal{P}_{n} \mid \phi_{n}(\exp X) \leq \alpha_{n}\right\},
$$

therefore (7) is equivalent to

$$
\begin{array}{ll}
\min & d(\log A, X) \\
\text { s.t. } & X \in \log \mathcal{P}_{n}, \\
& \phi_{n}(\exp X) \leq \alpha_{n},
\end{array}
$$

where elements above the main diagonal of $X$ are variables. The first constraint in (10) means that $X$ belongs to the subspace of skew-symmetric matrices. In this paper we show that the second, nonlinear inequality is a convex constraint in case of inconsistency indices $C R$ (Saaty 1980), $C M$ (Koczkodaj 1993; Duszak and Koczkodaj 1994) and CI (Peláez and Lamata, 2003).

Problem (8) can be rewritten in the same way as above:

$$
\begin{array}{ll}
\min & \alpha \\
\text { s.t. } & d(\log A, X) \leq K, \\
& X \in \log \mathcal{P}_{n}, \\
& \phi_{n}(\exp X) \leq \alpha,
\end{array}
$$

where $\alpha$ and elements above the main diagonal of $X$ are variables.

The objective function $d$ can be replaced by using the well-known "Big M" method. Assume that $M \geq 1$ is given as an upper bound of the values of the elements in $A \in \mathcal{P}_{n}$ and the computed $\hat{A} \in \mathcal{P}_{n}$ matrices, which is determined as the optimal solution of problems (7) and (8), i.e.,

$$
1 / M \leq a_{i j} \leq M, 1 / M \leq \hat{a}_{i j} \leq M, i, j=1, \ldots, n .
$$

We can find such an upper bound $M$ if we get a bounded interval by knowing the actual level of $\phi_{n}$, which contains at least one optimal solution of problems (7), and (8). 
On the other hand, if a theoretical upper bound $M$ is not given, then a reasonable bound $M$ is usually determined on the values of the pairwise comparison matrices in every specific problem. Constraint (12) can be described as

$$
A, \hat{A} \in[1 / M, M]^{n \times n}
$$

in matrix form, and if the condition (13) associated with $\hat{A}$ is attached to problems (7) and also (8), we get

$$
\begin{array}{ll}
\min & d(A, \hat{A}) \\
\text { s.t. } & \hat{A} \in \mathcal{A}_{n}\left(\phi_{n}, \alpha_{n}\right) \cap[1 / M, M]^{n \times n},
\end{array}
$$

and, respectively,

$$
\begin{array}{ll}
\min & \alpha \\
\text { s.t. } & d(A, \hat{A}) \leq K, \\
& \hat{A} \in \mathcal{A}_{n}\left(\phi_{n}, \alpha\right) \cap[1 / M, M]^{n \times n} .
\end{array}
$$

Introduce $\bar{M}=\log M$, problems (14) and (15) become equivalent to

$$
\begin{array}{ll}
\min & d(\log A, X) \\
\text { s.t. } & X \in \log \mathcal{P}_{n} \cap[-\bar{M}, \bar{M}]^{n \times n}, \\
& \phi_{n}(\exp X) \leq \alpha_{n},
\end{array}
$$

and

$$
\begin{array}{ll}
\min & \alpha \\
\text { s.t. } & d(\log A, X) \leq K, \\
& X \in \log \mathcal{P}_{n} \cap[-\bar{M}, \bar{M}]^{n \times n}, \\
& \phi_{n}(\exp X) \leq \alpha .
\end{array}
$$

in the logarithmic space.

The "Big M" method can be applied for (16) and (17). Let $\bar{A}=\log A$, and introduce binary variables $y_{i j} \in\{0,1\}, 1 \leq i<j \leq n$. Problem (16) can be altered by using $\bar{A} \in[-\bar{M}, \bar{M}]^{n \times n}$ into the following mixed 0-1 programming problem:

$$
\begin{array}{lll}
\min & \sum_{i=1}^{n-1} \sum_{j=i+1}^{n} y_{i j} & \\
\text { s.t. } & \phi_{n}(\exp X) \leq \alpha_{n}, & \\
& x_{i j}=-x_{j i}, & 1 \leq i \leq j \leq n, \\
& -\bar{M} \leq x_{i j} \leq \bar{M}, & 1 \leq i<j \leq n, \\
& -2 \bar{M} y_{i j} \leq x_{i j}-\bar{a}_{i j} \leq 2 \bar{M} y_{i j}, & 1 \leq i<j \leq n, \\
& y_{i j} \in\{0,1\}, & 1 \leq i<j \leq n .
\end{array}
$$


The optimal value of (18) gives the minimal number of the matrix elements above the main diagonal to be modified in order to achieve $\phi_{n} \leq \alpha_{n}$. In the optimal solution, $y_{i j}=1$ indicates the matrix elements that (and their reciprocal pairs) are modified, and $\exp x_{i j}$ gives a feasible value of these elements.

Problem (18) may have multiple optimal solutions with respect to the binary variables. If all of them are of interest, we list them one by one as follows. Assume that $L^{*}$ is the optimum value of the problem (18), $y_{i j}^{*}, 1 \leq i<j \leq n$, is an optimal solution and $I_{0}^{*}=\left\{(i, j) \mid y_{i j}^{*}=0,1 \leq i<\right.$ $j \leq n\}$. By adding the constraint

$$
\sum_{i=1}^{n-1} \sum_{j=i+1}^{n} y_{i j}=L^{*}
$$

to (18) we can ensure, that the optimal solutions of (18) can only be the feasible solutions of (18)-(19).

The addition of constraint

$$
\sum_{(i, j) \in I_{0}^{*}} y_{i j} \geq 1
$$

excludes the already known solution from further search. If problem (18)(19) -(20) has no feasible solution, then all optimal solutions of (18) have been found. Otherwise, each recently found optimal solution brings a constraint as (20), and resolve (18)-(19)-(20). The algorithm stops in a finite number of steps, resulting in all optimal solutions through binary variables (18).

Problem (17) can also be rewritten as in (18):

$$
\begin{array}{cll}
\min & \alpha & \\
\text { s.t. } & & \phi_{n}(\exp X) \leq \alpha, \\
& \sum_{i=1}^{n-1} \sum_{j=i+1}^{n} y_{i j} \leq K, & \\
& x_{i j}=-x_{j i}, & \\
& -\bar{M} \leq x_{i j} \leq \bar{M}, & 1 \leq i \leq j \leq n, \\
& -2 \bar{M} y_{i j} \leq x_{i j}-\bar{a}_{i j} \leq 2 \bar{M} y_{i j}, & 1 \leq i<j \leq n, \\
& y_{i j} \in\{0,1\}, & 1 \leq i<j \leq n,
\end{array}
$$

If $\phi_{n}(\exp X)$ is a convex function of the elements (above the main diagonal) of $X$, then the relaxations of (18) and (21) are convex optimization problems, consequently, (18) and (21) are mixed 0-1 convex problems. 


\section{Inconsistency index $C R$ of Saaty}

Saaty (1980) proposed to index the inconsistency of pairwise comparison matrix $A$ of size $n \times n$ by a positive linear transformation of its largest eigenvalue $\lambda_{\max }$. The normalized right eigenvector associated to $\lambda_{\max }$ also plays an important role, since it provides the estimation of the weights in the eigenvector method. However, in this paper weighting methods are not discussed. Saaty (1977) showed that $\lambda_{\max } \geq n$ and $\lambda_{\max }=n$ if and only if $A$ is consistent. Let us generate a large number of random pairwise comparison matrices of size $n \times n$, where each element above the main diagonal are chosen from the ratio scale $1 / 9,1 / 8,1 / 7, \ldots, 1 / 2,1,2, \ldots, 8,9$ with equal probability. Take the largest eigenvalue of each matrix and let $\overline{\lambda_{\max }}$ denote their average value.

Let $R I_{n}=\left(\overline{\lambda_{\max }}-n\right) /(n-1)$. Saaty defined the inconsistency of matrix $A$ as

$$
C R_{n}(A)=\frac{\frac{\lambda_{\max }(A)-n}{n-1}}{R I_{n}}
$$

being a positive linear transformation of $\lambda_{\max }(A)$. Then $C R_{n}(A) \geq 0$ and $C R_{n}(A)=0$ if and only if $A$ is consistent. The heuristic rule of acceptance is $C R_{n} \leq 0.1$ for all sizes, also known as the ten percent rule (Saaty, 1980), supported by Vargas' (1982) statistical analysis. However, some refinements are also known: $C R_{3} \leq 0.05$ for $3 \times 3$ matrices $C R_{4} \leq 0.08$ for $4 \times 4$ matrices (Saaty, 1994). Note that any rule of acceptance is somehow heuristic.

Now we apply the results of Section 2 by setting $\phi_{n}=C R_{n}$. Let $X \in \log \mathcal{P}_{n}$ and let $\lambda_{\max }(\exp X)$ denote the largest eigenvalue of $A=\exp X$. Then

$$
\phi_{n}(\exp X)=\frac{\lambda_{\max }(\exp X)-n}{R I_{n}(n-1)}
$$

Bozóki et al. (2010) showed that $\lambda_{\max }(\exp X)$ is a convex function of the elements of $X$, therefore, through (22),$\phi_{n}(\exp X)$ is a convex function of the elements of $X$, too.

It is proven that (22) implies that both (18) and (21) are mixed 0-1 convex optimization problems. However, they are still challenging from numerical computational point of view, since $\phi_{n}(\exp X)$ cannot be given in an explicit form as $\lambda_{\max }$ values are themselves computed by iterative methods (Saaty, 1980). We will show that $\lambda_{\max }$ is not only a limit of an iterative process, but an optimal solution of a convex optimization problem as well. The embedded convex optimization problem can be considered together the embedding optimization problem. 
Harker (1987) described the derivatives of $\lambda_{\max }$ with respect to a matrix element and recommended to change the element with the largest decrease in $\lambda_{\max }$. The theorems in this section, based on other tools, can be considered as some extensions of Harker's idea. Reducing $C R$, being equivalent to decreasing $\lambda_{\max }$, is in the focus of $\mathrm{Xu}$ and Wei (1999) and Cao et al. (2008).

A special case of Frobenius theorem is applied (Saaty, 1977; Sekitani and Yamaki, 1999):

Theorem 1. Let $A$ be an $n \times n$ irreducibile nonnegative matrix and $\lambda_{\max }(A)$ denote the maximal eigenvalue of $A$. Then the following equalities hold

$$
\max _{w>0} \min _{i=1, \ldots, n} \frac{\sum_{j=1}^{n} a_{i j} w_{j}}{w_{i}}=\lambda_{\max }(A)=\min _{w>0} \max _{i=1, \ldots, n} \frac{\sum_{j=1}^{n} a_{i j} w_{j}}{w_{i}} .
$$

Since the pairwise comparison matrices are positive, Theorem 1 can be applied. In order to rewrite the right-hand side of (23), $\bar{a}_{i j}=\log a_{i j}$, $i, j=1, \ldots, n$, and $z_{i}=\log w_{i}, i=1, \ldots, n$ are used:

$$
\lambda_{\max }(A)=\min _{z} \max _{i=1, \ldots, n} \sum_{j=1}^{n} e^{\bar{a}_{i j}+z_{j}-z_{i}}
$$

The sum of convex exponential functions in the right-hand side (24), furthermore, their maximum are also convex. Thus, $\lambda_{\max }$ can be determined as the optimum value of a convex optimization problem, and the form (24) is equivalent to the optimization problem

$$
\min \lambda \text { s.t. } \quad \sum_{j=1}^{n} e^{\bar{a}_{i j}+z_{j}-z_{i}} \leq \lambda, i=1, \ldots, n,
$$

where $\lambda$ and $z_{i}, i=1, \ldots, n$ are variables.

Let $\alpha_{n}$ be given as a threshold of inconsistency index $\phi_{n}=C R_{n}$. Then the constraint

$$
\phi_{n}(\exp X) \leq \alpha_{n}
$$

from problem (18) can be transformed by using (22) as

$$
\lambda_{\max }(\exp X) \leq n+R I_{n}(n-1) \alpha_{n} .
$$


Denote $\alpha_{n}^{*}=n+R I_{n}(n-1) \alpha_{n}$. Hence, the formula (24), substituting $x_{i j}=\bar{a}_{i j}$, implies an equivalent form

$$
\sum_{j=1}^{n} e^{x_{i j}+z_{j}-z_{i}} \leq \alpha_{n}^{*}, i=1, \ldots, n .
$$

Let us replace formula (26) by (28) in problem (18). We get a mixed 0-1 convex programming problem:

$$
\begin{array}{lll}
\min & \sum_{i=1}^{n-1} \sum_{j=i+1}^{n} y_{i j} & \\
\text { s.t. } & \sum_{j=1}^{n} e^{x_{i j}+z_{j}-z_{i}} \leq \alpha_{n}^{*}, & i=1, \ldots, n, \\
& x_{i j}=-x_{j i}, & 1 \leq i \leq j \leq n, \\
& -\bar{M} \leq x_{i j} \leq \bar{M}, & 1 \leq i<j \leq n, \\
& -2 \bar{M} y_{i j} \leq x_{i j}-\bar{a}_{i j} \leq 2 \bar{M} y_{i j}, & 1 \leq i<j \leq n, \\
& y_{i j} \in\{0,1\}, & 1 \leq i<j \leq n .
\end{array}
$$

Theorem 2. Let $\alpha_{n}$ denote the acceptance threshold of inconsistency and let $\alpha_{n}^{*}=n+R I_{n}(n-1) \alpha_{n}$. Then the optimum value of (29) gives the minimal number of the elements to be modified above the main diagonal in $A$ (and their reciprocals) in order to achieve that $C R_{n} \leq \alpha_{n}$.

Problem (21) can also be rewritten in case of $\phi_{n}=C R_{n}$. In the light of (22) , the minimization of $\phi_{n}$ is equivalent to the minimization of $\lambda_{\max }$. Furthermore, program (25) depending on $\lambda_{\max }$ is used to obtain:

$$
\begin{array}{lll}
\min & \lambda & \\
\text { s.t. } & \sum_{j=1}^{n} e^{x_{i j}+z_{j}-z_{i}} \leq \lambda, & \\
& & \\
& \sum_{i=1}^{n-1} \sum_{j=i+1}^{n} y_{i j} \leq K, & \\
& x_{i j}=-x_{j i}, & 1 \leq i \leq j \leq n, \\
& -\bar{M} \leq x_{i j} \leq \bar{M}, & 1 \leq i<j \leq n, \\
& -2 \bar{M} y_{i j} \leq x_{i j}-\bar{a}_{i j} \leq 2 \bar{M} y_{i j}, & 1 \leq i<j \leq n, \\
& y_{i j} \in\{0,1\}, & 1 \leq i<j \leq n .
\end{array}
$$

Theorem 3. Denote the optimum value of $(30)$ by $\lambda_{\mathrm{opt}}$, and let $\alpha_{\mathrm{opt}}=\frac{\lambda_{\mathrm{opt}}-n}{R I_{n}(n-1)}$. Then $\alpha_{\mathrm{opt}}$ is the minimal value of inconsistency $C R_{n}$ which can be obtained by the modification of at most $K$ elements above the main diagonal of $A$ (and their reciprocals). 


\section{Inconsistency index $C M$ of Koczkodaj and Duszak}

The inconsistency index introduced by Koczkodaj (1993) is based on $3 \times 3$ submatrices, called triads. For the $3 \times 3$ pairwise comparison matrix

$$
\left(\begin{array}{ccc}
1 & a & b \\
1 / a & 1 & c \\
1 / b & 1 / c & 1
\end{array}\right)
$$

let

$$
C M(a, b, c)=\min \left\{\frac{1}{a}\left|a-\frac{b}{c}\right|, \frac{1}{b}|b-a c|, \frac{1}{c}\left|c-\frac{b}{a}\right|\right\} .
$$

$C M$ can be extended to larger sizes (Duszak and Koczkodaj, 1994):

$$
C M(A)=\max \left\{C M\left(a_{i j}, a_{i k}, a_{j k}\right) \mid 1 \leq i<j<k \leq n\right\} .
$$

Unlike $C R_{n}$, the construction above does not contain any parameter depending on $n$, so we dispense with the use of the notation $C M_{n}$. It is easy to see that $C M$ is an inconsistency index since $C M(A) \geq 0$ for any $A \in \mathcal{P}_{n}$, and $C M(A)=0$ if and only if $A$ is consistent.

For a general triad $(a, b, c)$ let

$$
T(a, b, c)=\max \left\{\frac{a c}{b}, \frac{b}{a c}\right\} .
$$

It can be shown (Bozóki and Rapcsák, 2008) that there exists a direct relation between $C M$ and $T$ :

$$
C M(a, b, c)=1-\frac{1}{T(a, b, c)}, \quad T(a, b, c)=\frac{1}{1-C M(a, b, c)} .
$$

Since $T(a, b, c) \geq 1$, we get $0 \leq C M(a, b, c)<1$, so $0 \leq C M(A)<1$.

Let $(\bar{a}, \bar{b}, \bar{c})$ denote the logarithmized values of the triad $(a, b, c)$, and let

$$
\bar{T}(\bar{a}, \bar{b}, \bar{c})=\max \{\bar{a}+\bar{c}-\bar{b},-(\bar{a}+\bar{c}-\bar{b})\} .
$$

Then

$$
\begin{aligned}
T(a, b, c) & =\exp (\bar{T}(\bar{a}, \bar{b}, \bar{c})) \\
C M(a, b, c) & =1-\frac{1}{\exp (\bar{T}(\bar{a}, \bar{b}, \bar{c}))} .
\end{aligned}
$$


It is easy to check that even for triads, $C M$ is not a convex function of the logarithmized matrix elements, thus, if we choose the inconsistency index $\phi_{n}=C M$, then $\phi_{n}(\exp X)$ appearing in (18) and (21) is not a convex function of the element of matrix $X$. We show however that by using the univariate function

$$
f(t)=\frac{1}{1-t}
$$

being strictly monotone increasing on the interval $(-\infty, 1), f\left(\phi_{n}(\exp X)\right)=$ $f(C M(\exp X))$ is already a convex function of the elements of matrix $X$. Then we can change the constraint

$$
\phi_{n}(\exp X) \leq \alpha_{n}
$$

of problem (18) to the convex constraint

$$
f\left(\phi_{n}(\exp X)\right) \leq f\left(\alpha_{n}\right) .
$$

Also, instead of function $\phi_{n}(\exp X)$ appearing in problem (21) we can write $f\left(\phi_{n}(\exp X)\right)$ directly, and the value $f^{-1}\left(\alpha^{*}\right)$ computed from the optimal value $\alpha^{*}$ of the modified problem is the optimal value of the original problem (21).

To show the statement above, extend the index $T$ defined in (33) for arbitrary $n \times n$ pairwise comparison matrix $A$ :

$$
T(A)=\max \left\{T\left(a_{i j}, a_{i k}, a_{j k}\right) \mid 1 \leq i<j<k \leq n\right\} .
$$

According to (34), used there for triads, there is a strictly monotone increasing functional relationship between $C M$ and $T$. Consequently,

$$
C M(A)=1-\frac{1}{T(A)}=f^{-1}(T(A)), \quad T(A)=\frac{1}{1-C M(A)}=f(C M(A)),
$$

where $f$ is the function defined in (37).

By expressing $T$ in the logarithmized space, we get

$$
T(\exp X)=\max \left\{\max \left\{e^{x_{i j}+x_{j k}+x_{k i}}, e^{-x_{i j}-x_{j k}-x_{k i}}\right\} \mid 1 \leq i<j<k \leq n\right\} .
$$

Since on the right-hand-side of (40) the maximum of convex functions is taken, $T(\exp X)$ is convex function of the elements of matrix $X$. Consequently, if we choose the inconsistency index $\phi_{n}=C M$, then $f\left(\phi_{n}(\exp X)\right)$ 
is already a convex function, and the problems (18) and (21) modified as shown above are already convex mixed 0-1 optimization problems.

Although $C M(\exp X)$ is not convex, it is quasiconvex. To prove it, we show that the lower level sets of $C M(\exp X)$ are convex. Let $\beta \in[0,1)$ an arbitrary possible value of $C M(\exp X)$. Since $f$ is strictly monotone increasing, we have

$$
\left\{X \in \mathbb{R}^{n \times n} \mid C M(\exp X) \leq \beta\right\}=\left\{X \in \mathbb{R}^{n \times n} \mid f(C M(\exp X)) \leq f(\beta)\right\} .
$$

Due to the convexity of $T(\exp X)=f(C M(\exp X))$ the above level set are convex, and this implies the quasiconvexity of $C M(\exp X)$.

Theorem 4. $C M(\exp X)$ is quasiconvex on the set of the $n \times n$ matrices, and $T(\exp X)=f(C M(\exp X))$ is convex, where $f$ is defined in (37).

In the following we show that problems (18) and (21) can be solved in an easier way, namely, by solving appropriate linear mixed 0-1 optimization problems. By exploiting the strictly monotone increasing property of the exponential function, (40) can also be written in the following form:

$$
T(\exp X)=e^{\max \left\{\max \left\{x_{i j}+x_{j k}+x_{k i},-x_{i j}-x_{j k}-x_{i k}\right\} \mid 1 \leq i<j<k \leq n\right\}} .
$$

Now, (41) also means that $C M(A)$ can be obtained by determining the maximum of linear expressions of the elements of matrix $\bar{A}=\log A$ and by applying the exponential function and function $f$ once.

Theorem 5. (Bozóki et al. 2011a) For any $n \times n$ pairwise comparison matrix A, inconsistency index $C M$ can be obtained from the optimal solution of the following univariate linear program:

$$
\begin{array}{cll}
\min & z & \\
\mathrm{s.t.} & \bar{a}_{i j}+\bar{a}_{j k}+\bar{a}_{k i} \leq z, & 1 \leq i<j<k \leq n, \\
& -\left(\bar{a}_{i j}+\bar{a}_{j k}+\bar{a}_{k i}\right) \leq z & 1 \leq i<j<k \leq n .
\end{array}
$$

Let $z_{\mathrm{opt}}$ be the optimal value of (42). Then $C M(A)=1-\frac{1}{\exp \left(z_{\mathrm{opt}}\right)}$.

In the following let $\alpha_{n}$ denote the acceptance threshold associated with the inconsistency index $\phi_{n}=C M$, and let

$$
\alpha_{n}^{*}=\log \left(\frac{1}{1-\alpha_{n}}\right) \text {. }
$$


Consider the linear mixed 0-1 optimization problem

$$
\begin{array}{lll}
\min & \sum_{i=1}^{n-1} \sum_{j=i+1}^{n} y_{i j} & \\
\text { s.t. } & x_{i j}+x_{j k}+x_{k i} \leq \alpha_{n}^{*}, & 1 \leq i<j<k \leq n, \\
& -\left(x_{i j}+x_{j k}+x_{k i}\right) \leq \alpha_{n}^{*}, & 1 \leq i<j<k \leq n, \\
& x_{i j}=-x_{j i}, & 1 \leq i \leq j \leq n, \\
& -\bar{M} \leq x_{i j} \leq \bar{M}, & 1 \leq i<j \leq n, \\
& -2 \bar{M} y_{i j} \leq x_{i j}-\bar{a}_{i j} \leq 2 \bar{M} y_{i j}, & 1 \leq i<j \leq n, \\
& y_{i j} \in\{0,1\}, & 1 \leq i<j \leq n .
\end{array}
$$

Based on the findings above, the following two theorems follow.

Theorem 6. Let $\alpha_{n}$ denote the acceptance threshold of inconsistency and let $\alpha_{n}^{*}=\log \left(\frac{1}{1-\alpha_{n}}\right)$. Then the optimum value of (44) gives the minimal number of the elements to be modified above the main diagonal in $A$ (and their reciprocals) in order to achieve that $C M \leq \alpha_{n}$.

By some alterations in (44), the following linear mixed 0-1 optimization problem can be written:

$$
\begin{array}{cll}
\min & \alpha & \\
\mathrm{s.t.} & & x_{i j}+x_{j k}+x_{k i} \leq \alpha, \\
& -\left(x_{i j}+x_{j k}+x_{k i}\right) \leq \alpha, & 1 \leq i<j<k \leq n, \\
& \sum_{i=1}^{n-1} \sum_{j=i+1}^{n} y_{i j} \leq K, & \\
& x_{i j}=-x_{j i}, & \\
& -\bar{M} \leq x_{i j} \leq \bar{M}, & 1 \leq i \leq j \leq n, \\
& -2 \bar{M} y_{i j} \leq x_{i j}-\bar{a}_{i j} \leq 2 \bar{M} y_{i j}, & 1 \leq i<j \leq n, \\
& y_{i j} \in\{0,1\}, & 1 \leq i<j \leq n,
\end{array}
$$

Theorem 7. Let $\alpha_{\mathrm{opt}}$ denote the optimum value of $\sqrt{45)}$. Then $1-\frac{1}{\exp \left(\alpha_{\mathrm{opt}}\right)}$ is the minimal value of inconsistency $C M$ which can be obtained by the modification of at most $K$ elements above the main diagonal of $A$ (and their reciprocals).

\section{Inconsistency index $C I$ of Peláez and Lamata}

Similarly to $C M$, the inconsistency index $C I$ proposed by Peláez and Lamata (2003) is also based on triads of form (31). It is easy to see that the determinant of the triad (31) is nonnegative, and it is zero if and only if the triad 
is consistent. Based on this interesting property, Peláez and Lamata (2003) proposed to characterize the inconsistency of a pairwise comparison matrix $A \in \mathcal{P}_{n}$ by the average of the determinants of the triads of matrix $A$ :

$$
C I_{n}(A)= \begin{cases}\operatorname{det}(A), & \text { for } n=3, \\ \frac{1}{N T(n)} \sum_{i=1}^{N T(n)} \operatorname{det}\left(\Gamma_{i}\right), & \text { for } n>3\end{cases}
$$

where $\Gamma_{i}, i=1, \ldots, N T(n)$ denote the triads of matrix $A$, and $N T(n)=\left(\begin{array}{l}n \\ 3\end{array}\right)$ is the number of triads in $A$.

We show that $C I$ is a convex function of the logarithmized matrix elements, thus if the inconsistency index $\phi_{n}=C I_{n}$ is chosen, then $\phi_{n}(\exp X)$ appearing in problems (18) and (21) is a convex function of the elements of matrix $X$.

The determinant of triad $\Gamma \in \mathcal{P}_{3}$ comparing objects $(i, j, k)$ can be written as

$$
\operatorname{det}(\Gamma)=\frac{a_{i k}}{a_{i j} a_{j k}}+\frac{a_{i j} a_{j k}}{a_{i k}}-2 .
$$

Let $X=\log \Gamma \in \log \mathcal{P}_{3}$, i.e., $\Gamma=\exp X$. Equation (47) can be reformulated as a convex function of the elements of $X$ :

$$
\operatorname{det}(\exp X)=e^{x_{i k}-x_{i j}-x_{j k}}+e^{x_{i j}+x_{j k}-x_{i k}}-2 .
$$

Let $\alpha_{n}$ be a given acceptance threshold for the inconsistency index $\phi_{n}=C I_{n}$. According to (46) and (48), the constraint

$$
\phi_{n}(\exp X) \leq \alpha_{n}
$$

appearing in (18) can be expressed as

$$
\frac{1}{\left(\begin{array}{l}
n \\
3
\end{array}\right)} \sum_{i=1}^{n-2} \sum_{j=i+1}^{n-1} \sum_{k=j+1}^{n}\left(e^{x_{i k}-x_{i j}-x_{j k}}+e^{x_{i j}+x_{j k}-x_{i k}}-2\right) \leq \alpha_{n} .
$$

By denoting $\alpha_{n}^{*}=\left(\alpha_{n}+2\right)\left(\begin{array}{l}n \\ 3\end{array}\right)$, (50) can be simplified as

$$
\sum_{i=1}^{n-2} \sum_{j=i+1}^{n-1} \sum_{k=j+1}^{n}\left(e^{x_{i k}-x_{i j}-x_{j k}}+e^{x_{i j}+x_{j k}-x_{i k}}\right) \leq \alpha_{n}^{*}
$$


and inserting it into (18), we get the mixed 0-1 convex optimization problem

$$
\begin{aligned}
& \min \sum_{i=1}^{n-1} \sum_{j=i+1}^{n} y_{i j} \\
& \text { s.t. } \quad \sum_{i=1}^{n-2} \sum_{j=i+1}^{n-1} \sum_{k=j+1}^{n}\left(e^{x_{i k}-x_{i j}-x_{j k}}+e^{x_{i j}+x_{j k}-x_{i k}}\right) \leq \alpha_{n}^{*} \text {, } \\
& x_{i j}=-x_{j i}, \quad 1 \leq i<j \leq n, \\
& -\bar{M} \leq x_{i j} \leq \bar{M}, \quad 1 \leq i<j \leq n, \\
& -2 \bar{M} y_{i j} \leq x_{i j}-\bar{a}_{i j} \leq 2 \bar{M} y_{i j}, \quad 1 \leq i<j \leq n, \\
& y_{i j} \in\{0,1\}, \quad 1 \leq i<j \leq n \text {. }
\end{aligned}
$$

Theorem 8. Let $\alpha_{n}$ denote the acceptance threshold of inconsistency and let $\alpha_{n}^{*}=\left(\alpha_{n}+2\right)\left(\begin{array}{l}n \\ 3\end{array}\right)$. Then the optimum value of (52) gives the minimal number of the elements to be modified above the main diagonal in $A$ (and their reciprocals) in order to achieve that $C I \leq \alpha_{n}$.

In the same way as for other inconsistency indices, the following mixed 0-1 convex optimization problem can also be considered:

$$
\begin{aligned}
& \min \quad \alpha \\
& \text { s.t. } \quad \sum_{i=1}^{n-2} \sum_{j=i+1}^{n-1} \sum_{k=j+1}^{n}\left(e^{x_{i k}-x_{i j}-x_{j k}}+e^{x_{i j}+x_{j k}-x_{i k}}\right) \leq \alpha \text {, } \\
& x_{i j}=-x_{j i}, \quad 1 \leq i<j \leq n, \\
& -\bar{M} \leq x_{i j} \leq \bar{M}, \quad 1 \leq i<j \leq n, \\
& -2 \bar{M} y_{i j} \leq \bar{x}_{i j}-\bar{a}_{i j} \leq 2 \bar{M} y_{i j}, \quad 1 \leq i<j \leq n, \\
& y_{i j} \in\{0,1\}, \quad 1 \leq i<j \leq n, \\
& \sum_{i=1}^{n-1} \sum_{j=i+1}^{n} y_{i j} \leq K \text {. }
\end{aligned}
$$

Theorem 9. Let $\alpha_{\mathrm{opt}}$ denote the optimum value of (53). Then $\frac{\alpha_{\mathrm{opt}}}{\left(\begin{array}{c}n \\ 3\end{array}\right)}-2$ is the minimal value of inconsistency $C I$ which can be obtained by the modification of at most $K$ elements above the main diagonal of $A$ (and their reciprocals).

\section{A numerical example}

Our approach is also presented on a classic numerical example from the book of Saaty (1980), for the inconsistency index $C R$. Table 1 contains pairwise comparison values of six cities concerning their distances from Philadelphia. As an example, the evaluator judged that the distance between London and 
Philadelphia is five times greater than that between Chicago and Philadelphia.

Table 1. Comparison of distances of cities from Philadelphia

\begin{tabular}{lllllll}
\hline & Cairo & Tokyo & Chicago & San Francisco & London & Montreal \\
\hline Cairo & 1 & $1 / 3$ & 8 & 3 & 3 & 7 \\
Tokyo & 3 & 1 & 9 & 3 & 3 & 9 \\
Chicago & $1 / 8$ & $1 / 9$ & 1 & $1 / 6$ & $1 / 5$ & 2 \\
San Francisco & $1 / 3$ & $1 / 3$ & 6 & 1 & $1 / 3$ & 6 \\
London & $1 / 3$ & $1 / 3$ & 5 & 3 & 1 & 6 \\
Montreal & $1 / 7$ & $1 / 9$ & $1 / 2$ & $1 / 6$ & $1 / 6$ & 1 \\
\hline
\end{tabular}

Let $A$ denote the pairwise comparison matrix concerning Table 1 . We get that $\lambda_{\max }(A)=6.4536$, and from $R I_{6}=1.24$, also $C R(A)=0.0732$. Since the value of $C R(A)$ is significantly below the $10 \%$ threshold, we can consider the inconsistency of $A$ acceptable.

Let $A^{(1)}$ denote the matrix obtained from $A$ by exchanging the elements $a_{1,2}$ (and $a_{2,1}$ ). This is a typical mistake at filling-in a pairwise comparison matrix. For the matrix $A^{(1)}$, we get $C R\left(A^{(1)}\right)=0.0811$. Therefore, although the level of inconsistency of $A^{(1)}$ has increased as consequence of the datarecording error, it is still below the acceptance level of $10 \%$. In this case the proposed methodology is not able to detect the mistake, and $A^{(1)}$ is still accepted.

Consider now the case when $a_{1,3}$ and $a_{3,1}$ are exchanged, say by accident, in the matrix $A$. Let $A^{(2)}$ denote the matrix obtained in this way. Then $C R\left(A^{(2)}\right)=0.5800$, which is well over the acceptance level of $10 \%$, and it refers to a rough inconsistency in the matrix. By solving the corresponding problem (29), we obtain that the inconsistency of $A^{(2)}$ can be pushed below the critical $10 \%$ by modifying a single element (and its reciprocal). This element is just in the spoilt position $a_{1,3}$. It can also be shown that this is the single optimal solution to problem (29) considering the 0-1 variables. Consequently, the proposed methodology has detected the single possible element for the case of correcting in a single position (and in its reciprocal). It also turned out that this single position is just the one of the values exchanged by accident.

In the previous example the spoilt matrix caused a rough increase of the inconsistency. In this view, it is not surprising that the proposed method offers a unique way of repairing. However, at smaller increase of inconsistency the situation is not that obvious. 
Assume now that the element $a_{1,3}$ of matrix $A$ is changed to 2 instead of the value $1 / 8$ of the previous example. This is a smaller difference in relation to the original value 8 , the increase of the inconsistency of the modified matrix, denoted by $A^{(3)}$, is also less: $C R\left(A^{(3)}\right)=0.1078$. The inconsistency of $A^{(3)}$ barely exceeds the critical level $10 \%$, therefore, one would expect that by the modification of a single element can make the inconsistency decrease below 10\%, and also that several positions are eligible for this purpose. Indeed, the optimal value of the relating problem (29) is 1 , and by resolving the problem after adding the constraints (19) and (20) we find that problem (29) has 6 different optimal solutions according to the binary variables. Namely, the inconsistency of matrix $A^{(3)}$ decreases below $10 \%$ not only by modifying $a_{1,3}$, but also by modifying any single element of $\left\{a_{1,4}, a_{1,5}, a_{2,6}, a_{3,4}, a_{4,5}\right\}$. In the ideal case, the evaluator spots the data-recording error in position $a_{1,3}$ immediately. If not, then $\mathrm{s} /$ he may have to reconsider the evaluation of each of the 6 positions, but it is still fewer than the 15 possible positions in the upper triangular part of the matrix.

\section{References}

Bozóki S, Fülöp J, Koczkodaj WW (2011a) LP-based consistency-driven supervision for incomplete pairwise comparison matrices. Mathematical and Computer Modelling 54(1-2):789-793

Bozóki S, Fülöp J, Poesz A (2011b) On pairwise comparison matrices that can be made consistent by the modification of a few elements. Central European Journal of Operations Research 19(2):157-175

Bozóki S, Fülöp J, Poesz A (2012) Convexity properties related to pairwise comparison matrices of acceptable inconsistency and applications, (in Hungarian, Elfogadható inkonzisztenciájú páros összehasonlítás mátrixokkal kapcsolatos konvexitási tulajdonságok és azok alkalmazásai). In: Solymosi T, Temesi J (eds.) Egyensúly és optimum: Tanulmányok Forgó Ferenc 70. születésnapjára, Aula Kiadó, pp. 169-184

Bozóki S, Fülöp J, Rónyai L (2010) On optimal completions of incomplete pairwise comparison matrices. Mathematical and Computer Modelling 52(1-2):318-333

Bozóki S, Rapcsák T (2008) On Saaty's and Koczkodaj's inconsistencies of pairwise comparison matrices. Journal of Global Optimization 42(2):157175

Brunelli M, Fedrizzi M (2011) Characterizing properties for inconsistency indices in the AHP. Proceedings of the 11th International Symposium on the AHP, Sorrento, Naples, Italy, June 15-18, 2011 
Brunelli M, Fedrizzi M (2013a) Axiomatic properties of inconsistency indices for pairwise comparisons. Submitted, http://arxiv.org/abs/1306.6852

Brunelli M, Canal L, Fedrizzi M (2013b) Inconsistency indices for pairwise comparison matrices: a numerical study. Annals of Operations Research, published online first, DOI 10.1007/s10479-013-1329-0

Cao D, Leung LC, Law JS (2008) Modifying inconsistent comparison matrix in analytic hierarchy process: A heuristic approach. Decision Support Systems 44(4):944-953

Chu MT (1997) On the optimal consistent approximation to pairwise comparison matrices. Linear Algebra and its Applications 272(1-3):155-168

Duszak Z, Koczkodaj WW (1994) Generalization of a new definition of consistency for pairwise comparisons. Information Processing Letters 52(5):273-276

Harker PT (1987) Derivatives of the Perron root of a positive reciprocal matrix: With application to the analytic hierarchy process. Applied Mathematics and Computation 22(2-3):217-232

Koczkodaj WW (1993) A new definition of consistency of pairwise comparisons. Mathematical and Computer Modelling 18(7):79-84

Peláez JI, Lamata MT (2003) A new measure of consistency for positive reciprocal matrices. Computers and Mathematics with Applications 46:18391845

Saaty TL (1977) A scaling method for priorities in hierarchical structures. Journal of Mathematical Psychology 15(3):234-281

Saaty TL (1980) The Analytic Hierarchy Process, McGraw-Hill, New York

Saaty TL (1994) Fundamentals of Decision Making, RSW Publications, Pittsburgh

Sekitani K, Yamaki N (1999) A logical interpretation for the eigenvalue method in AHP. Journal of the Operations Research Society of Japan 42(2):219-232

Vargas LG (1982) Reciprocal matrices with random coefficients. Mathematical Modelling 3(1):69-81

Xu Z, Wei C (1999) A consistency improving method in the analytic hierarchy process. European Journal of Operational Research 116(2):443-449 